Prior Learning and the Efficacy of Retrieval Practice

$$
\begin{gathered}
\text { Mohan W. Gupta }{ }^{1} \text {, Steven C. Pan }{ }^{2}, \text { \& Timothy C. Rickard }{ }^{1} \\
{ }^{1} \text { University of California, San Diego } \\
{ }^{2} \text { University of California, Los Angeles }
\end{gathered}
$$

\footnotetext{
Author note

Correspondence concerning this article should be addressed to Timothy C. Rickard, Department of Psychology, University of California, San Diego, La Jolla, Ca. 92093-0109. E-mail: trickard@ucsd.edu.
} 


\begin{abstract}
In three experiments we investigated how the level of study-based learning influences the efficacy of subsequent retrieval practice (testing). Possibilities are that the efficacy of a test relative to a restudy control decreases, increases, or is independent of the degree of prior learning. Prior learning was manipulated by varying the number of item repetitions in the initial study phase between one and eight. Predictions of the dual-memory model for the testing effect were used as a reference for inference. Results support the hypothesis that the advantage of testing over restudy is independent of the degree of prior learning. Those results can serve to constrain future theory development, and they suggest that educators can apply cued-recall testing with the expectation that its efficacy is similar across varying levels of prior content knowledge.
\end{abstract}

Keywords: cued-recall; testing effect; retrieval practice; dual-memory model; prior knowledge; individual differences 


\section{Prior Learning and the Efficacy of Retrieval Practice}

Extensive research has established that, over a broad range of conditions and populations, some learning methods are more effective than others. Educationally relevant examples include spaced practice (Cepeda et al., 2006; Cepeda et al., 2008; Mozer et al., 2009; Carpenter et al., 2012; Rohrer, 2015) and interleaved practice (Rohrer et al., 2012; Pan et al., 2019; Foster et al., 2019; Rohrer et al., 2020). A third finding in that vein, and the one on which we focus in the current paper, is that retrieval from memory can produce better learning and retention than a non retrieval-based learning activity, such as passive study. That result has been obtained over a variety of materials and settings (for reviews see Karpicke, Lehman, and Aue, 2014; Kornell and Vaughn, 2016; Rickard \& Pan, 2018; Roediger \& Karpicke, 2006; Rowland, 2014; van den Broek et al., 2016). For example, answering the question, "what is the powerhouse of the cell?" yields a higher probability of retrieving "mitochondria" on a later test than does studying "the powerhouse of the cell is the mitochondria," particularly if correct answer feedback is provided after each question (Pashler et al., 2005; Kang, McDermott, and Roediger, 2007). On the final test in such studies, the proportion correct for items in the test condition, minus the proportion correct for items in the restudy condition, is variously known as the retrieval practice effect, the test-enhanced learning effect, and the testing effect.

The testing effect has been observed for multiple test types, but most frequently cued-recall. A typical cued-recall testing effect paradigm involves two sessions separated by a retention interval: the study and training phases are conducted during session 1 and the final test during session 2. During the study phase, materials such as facts or word-pairs are studied (e.g. lime-salt). During the training phase half of the materials are restudied and half are tested (e.g. lime-?). The experiments of the current paper involve word-pair materials, and correct answer 
feedback (henceforth, feedback) is provided after each training phase test trial. During the final test in session 2, a cued-recall test is administered for all items.

For the case of cued-recall, a positive testing effect has been observed in about $96 \%$ of published experiments (Rickard \& Pan, 2018). The testing effect has been observed not only in the laboratory, but also in multiple applied settings, including medical resident classroom learning (Larsen et al., 2009), medical skill learning (Kronman et al., 2009), college classrooms using clickers (Lantz, 2010), children's spelling (Pan, Rubin, and Rickard, 2015), high school classrooms (Nungester, 1982), and university classrooms (McDaniel Roediger \& McDermott, 2007).

The testing effect paradigm lends itself to multiple theoretically and practically informative variations. Manipulations that have been explored in the literature include test type (Carpenter \& DeLosh, 2005), material type (Roediger \& Karpicke, 2006; Kronman et al., 2009; Pan, Rubin, and Rickard, 2015), presence or absence of feedback (Kang, McDermott, and Roediger, 2007), retention interval (Carpenter, Pashler, Wixted, and Vul, 2008; Kornell, Bjork, and Garcia, 2011), and blocked vs. random sequencing of test and restudy trials during training (Abel \& Roediger, 2017), among many others. To our knowledge, one basic aspect of that paradigm that has yet to be manipulated is the degree of learning that is achieved during the study phase, and its effects on the efficacy of subsequent testing. From an applied perspective, it is of value to know if and how the benefits of testing for memory and retention are moderated by prior learning. Consider two students who are entering an introductory college biology course, only one of which has taken an Advanced Placement biology course in high school whereas the other student took regular biology class. Is retrieval practice equally effective in promoting new learning for those two students? As another example that is more analogous to the current study, 
some students are likely to have studied their notes or read a book chapter once prior to a test, or quiz, whereas other students may have done so two or more times, yielding increased knowledge of the material prior to the quiz. Is the quiz's effectiveness for new learning moderated by that difference in prior study-based knowledge? We explore that question in the current paper by manipulating the number of study phase item repetitions in a cued-recall paradigm, with the expectation that study phase learning will increase monotonically with increasing repetition. Over three experiments, each condition involved either one study phase trial per item (1x study repetition), four study phase trials per item (4x study repetitions), or eight study trials per item (8x study repetitions). Across all experiments, we held training phase exposure constant at one trial per item in both the test and restudy conditions.

The question of how study-based learning influences learning on a subsequent test is pertinent to theoretical accounts of the testing effect. One possibility is that increasing study-based learning, while holding the training phase exposure constant, will decrease the efficacy of testing relative to restudy on the final test. We will refer to that possibly as the attenuation hypothesis. The only theory to date that makes predictions about the effect of study phase learning on the testing effect - the dual-memory model of Rickard \& Pan (2018) - is consistent with the attenuation hypothesis. That theory is described in the next section. An empirical phenomenon that is potentially consistent with attenuation is the pretesting effect (Richland et al., 2009), in which a test with feedback trial can yield substantially more learning than does a time-equated study trial, even though there is no prior study. The final test performance advantage for pretesting can be as large as or larger than that in the testing effect paradigm (Pan \& Sana, 2021; cf. Latimier et al., 2019). In the current terminology, pretesting constitutes the extreme case $0 x$ study phase repetition. 
Alternatively, increased learning through study repetition may not attenuate the effect of a test, but rather enhance it (i.e., the enhancement hypothesis). That hypothesis might hold, for example, (1) if more study phase repetition yields more learning, as expected, (2) more study phase learning yields a higher proportion correct on the training test, as expected, and (3) learning through that test is greater when the correct answer is retrieved from memory than when it is provided through feedback. In that scenario, the learning advantage of a training phase test vs. restudy should be enhanced with increased study phase repetition. Speaking against that possibility, there is evidence that training phase test accuracy does not causally influence final test performance when there is feedback on the training test (Kornell, Klein, \& Rawson, 2015; Rickard, 2020), although in our view that evidence is equivocal. The enhancement hypothesis could also hold even if learning on a test trial with feedback is not causally influenced by response accuracy. In general terms, a test may in some unknown way interact "synergistically" with the degree of learning that is acquired through prior study. A third possibility is that the amount of study phase learning neither attenuates nor enhances (i.e., is independent of) testing efficacy relative to restudy (the no effect hypothesis).

The hypotheses outlined above are best understood in terms of relative underlying memory strength for restudied and tested items. However, the dependent measure in the current experiments, and in the vast majority of the literature, is proportion correct. More specifically, it is the difference in final test proportion correct in the test and restudy conditions (i.e., the testing effect, or $T E$ ). Therefore, ceiling effects are a potential issue with data interpretation on this topic. For example, as the proportion correct in the restudy condition approaches one, the maximum TE magnitude must drop toward zero. That fact creates a confound if the final test restudy proportion correct increases to a high level with increasing study phase repetition and if 
the TE is observed to decrease with increasing study repetition. In that case, it would not be possible to differentiate between the process-based attenuation account described above and a mere ceiling effect on the TE.

In the current experiments we used two approaches to address that issue. First, the experimental designs were similar in most respects to those in our prior work on this topic, in which restudy proportion correct on the final test rarely exceeded .5 , reducing the possibility of a ceiling effect confound. Second and more decisively, we used the proportion correct prediction of the dual-memory model (Rickard \& Pan, 2018) for the case of $1 \mathrm{x}$ study repetition as a reference prediction not just for the $1 \mathrm{x}$ study groups of the current experiments - the case for which that model was originally intended to apply - but also for the $4 \mathrm{x}$ and 8 study groups. The logic behind that approach is that the dual-memory prediction for the 1x study case has proven highly accurate over multiple $1 \mathrm{x}$ study datasets. As we elaborate below, if the model prediction designed for the $1 \mathrm{x}$ repetition case holds for all study groups in the current experiments, then the no effect hypothesis will be supported. If the TE magnitude in the $4 \mathrm{x}$ and $8 \mathrm{x}$ study groups is smaller than that model predicts for the $1 \mathrm{x}$ study case, then the attenuation hypothesis will be supported. If the TE magnitude is larger than the model prediction for the $1 \mathrm{x}$ case, then the enhancement hypothesis will be supported. That approach does not require direct comparison between testing effect magnitudes at different study repetition levels, and thus it circumvents potential issues with proportion correct scaling and ceiling effects.

\section{The Dual Memory Model of Test Enhanced Learning}

The dual memory theoretical framework and the corresponding quantitative model is described in detail in Rickard and Pan (2018; see also Rickard, 2020; for application of the model to transfer of test enhanced learning, see Rickard \& Pan, 2020). Here we provide a brief 
description, specify the quantitative predictions of the model, and summarize the results of prior data fits.

That model makes predictions based on the simple premise that the study phase encodes a study memory and that study repetition (in both the study phase and through training phase restudy) strengthens (i.e., promotes later correct retrieval through) that study memory. A training phase test such as cued-recall with feedback, however, both strengthens study memory and encodes a new test memory, which provides a second and independent route to correct answer retrieval on the final test. Under the simplifying assumptions that, for each participant, (1) study memory strength after training is about the same for studied and tested items, and (2) study and test memory strengths for tested items are at least partially independent, then the chance of correctly remembering the target-word on the final test is higher in the test condition, creating the testing effect. Given several additional assumptions (Rickard and Pan, 2018), that theory was framed into a simplest case quantitative model.

Rickard and Pan (2018) described the dual memory quantitative model predictions for the case of no feedback using simulation. For the case of correct answer feedback after each trial, as in all of the current experiments, Rickard and Pan described a probability equation for a hypothetical ideal participant, and a corresponding equation that expresses model predictions for observed test condition proportion correct in the test condition for each experimental participant. In the probability model, $\mathrm{P}_{\mathrm{R}}$ for a given participant denotes the probability correct on the final test for a randomly selected item in the restudy condition is, $\mathrm{P}_{\mathrm{T}-\mathrm{s}}$ is the probability correct on the final test through study memory for a randomly selected item in the test condition, and $\mathrm{P}_{\mathrm{T}-\mathrm{t}}$ is the probability of retrieval through the test memory for a randomly selected item in the test condition. $\mathrm{P}_{\mathrm{T}}$ is the overall probability of a correct response for a randomly selected item in the 
test condition. The prediction for $\mathrm{P}_{\mathrm{T}}$ is based on the probability correct through either $\mathrm{P}_{\mathrm{T}-\mathrm{s}}$ (study memory), $\mathrm{P}_{\mathrm{T}-\mathrm{t}}$ (test memory), or both, governed by the product rule for independent events (given that strengths of study and test memory for tested items are assumed to be independent in the model):

$$
\mathrm{P}_{\mathrm{T}}=\mathrm{P}_{\mathrm{T}-\mathrm{s}}+\mathrm{P}_{\mathrm{T}-\mathrm{t}}-\mathrm{P}_{\mathrm{T}-\mathrm{S}} \mathrm{P}_{\mathrm{T}-\mathrm{t}} .
$$

Because $\mathrm{P}_{\mathrm{R}}=\mathrm{P}_{\mathrm{T}-\mathrm{s}}=\mathrm{P}_{\mathrm{T}-\mathrm{t}}$ in this simplest case model (see Rickard \& Pan, 2018), Equation 1 can be reduced to,

$$
\mathrm{P}_{\mathrm{T}}=\mathrm{P}_{\mathrm{R}}+\mathrm{P}_{\mathrm{R}}-\mathrm{P}_{\mathrm{R}} * \mathrm{P}_{\mathrm{R}}=2 \mathrm{P}_{\mathrm{R}}-\mathrm{P}_{\mathrm{R}}^{2}
$$

and the equation for the TE is,

$$
\mathrm{TE}=\mathrm{P}_{\mathrm{T}}-\mathrm{P}_{\mathrm{R}}=\mathrm{P}_{\mathrm{R}}-\mathrm{P}_{\mathrm{R}}^{2} \text {. }
$$

Hence, the model predicts that both probability correct in the test condition $\left(\mathrm{P}_{\mathrm{T}}\right)$ and the testing effect magnitude are solely a function of probability correct in the restudy condition, $\mathrm{P}_{\mathrm{R}}$.

For an actual participant in an experiment, the same quadratic equations describe the model's expected value prediction for the observed proportion correct in the test condition, $\mathrm{PC}_{\mathrm{T}}$, and for the TE, where $\mathrm{PC}_{\mathrm{R}}$ is the observed proportion correct in the restudy condition,

$$
\begin{aligned}
& \mathrm{PC}_{\mathrm{T}} \text {-predicted }=2 \mathrm{PC}_{\mathrm{R}}-\mathrm{PC}_{\mathrm{R}}{ }^{2}, \\
& \text { TE-predicted }=\mathrm{PC}_{\mathrm{R}}-\mathrm{PC}_{\mathrm{R}}{ }^{2} .
\end{aligned}
$$

The dual-memory model has no free parameters, is fitted individually for each participant, and makes interval scale predictions.

For the experimental paradigm outlined above with 1x study repetition, the predictions of Equation 4 (and hence, Equation 5) have held to a very close approximation over multiple datasets (Rickard \& Pan, 2018). Those experiments differed over multiple factors, including the mean restudy proportion correct, the retention interval between training and final test phases ( 5 
minutes to 7 day), and materials (paired associates, word-triplets, and history facts). The dual-memory model has provided close data fits not only for the mean $\mathrm{PC}_{\mathrm{T}}$ and the $\mathrm{TE}$, but also for the cumulative $\mathrm{PC}_{\mathrm{T}}$ distribution (Rickard, 2020). That result has been demonstrated across the retention intervals and materials variations noted above, still with no free parameters. Because the distribution of proportion correct scores in the TE paradigm clearly reflects not just sampling variability, but also to a large extent individual differences in task ability, those good distribution fits of Equation 4 indicate that the model accommodated participant individual differences that were relevant to performance in those experiments (Rickard, 2020).

Equation 4 was intended for the case of $1 \mathrm{x}$ study per item, the only study phase case to which the dual-memory model has been applied to date, and by far the most common study design in the literature. In that case, the simplifying assumption in the model that $\mathrm{P}_{\mathrm{R}}=\mathrm{P}_{\mathrm{T}-\mathrm{s}}=\mathrm{P}_{\mathrm{T}-\mathrm{t}}$ is plausible. However, given that study memory and test memory are assumed to be independent in that model, that equation cannot be mechanistically correct for both the $1 \mathrm{x}$ study repetition case and the multiple study phase repetition case (provided that learning increases with study repetition). The assumption that $\mathrm{P}_{\mathrm{R}}=\mathrm{P}_{\mathrm{T}-\mathrm{s}}$ remains viable in that case, because the number of study phase exposures remains the same for items that are assigned to the training phase restudy and test conditions. However, because of the independence assumption, test memory strength would not increase with increasing study repetitions. Rather, test memory strength will remain constant as the number of study phase repetitions increases. Hence, the model predicts a different relation between the three probabilities when there are multiple study phase repetitions:

$$
\mathrm{P}_{\mathrm{R}}=\mathrm{P}_{\mathrm{T}-\mathrm{s}}>\mathrm{P}_{\mathrm{T}-\mathrm{t}} .
$$

As an example, consider an experimental group in which there are $4 \mathrm{x}$ study repetitions. Also assume that probability correct through study memory for a given participant on the final 
test is, arbitrarily, .5, both for restudied items $\left(\mathrm{P}_{\mathrm{R}}\right)$ and tested items $\left(\mathrm{P}_{\mathrm{T}-\mathrm{s}}\right)$. Because of the assumed independence of test memory and the multiple study repetitions, however, the probably correct through test memory for tested items $\left(\mathrm{P}_{\mathrm{T}-\mathrm{t}}\right)$ is predicted to be lower (e.g., .3). Plugging those numbers into Equation 1 yields:

$$
\begin{aligned}
& \mathrm{P}_{\mathrm{T}}=.5+.3-(.5)(.3)=.65, \text { and predicted testing effect magnitude would be, } \\
& \mathrm{TE}=.65-.5=.15
\end{aligned}
$$

For comparison, consider an experiment in which there is only one study phase repetition and in which $\mathrm{P}_{\mathrm{R}}$ on the final test is again .5 (e.g., in a 1x study experimental design that is otherwise identical to that of the above example, but with the exception of a shorter retention interval, less forgetting, and hence larger $\left.\mathrm{P}_{\mathrm{R}}\right)$. The correct model prediction for that case is,

$$
\mathrm{P}_{\mathrm{T}}=.5+.5-(.5)(.5)=.75 \text {, and the predicted testing effect magnitude is, }
$$$$
\mathrm{TE}=.75-.5=.25 \text {. }
$$

Hence, the dual-memory model is consistent with the attenuation hypothesis. That is, holding the final test restudy proportion correct constant (e.g., at .5, as in two examples above), the model predicts that the testing effect magnitude will decrease with increasing study phase repetitions (e.g., .15 versus .25 in the examples above).

In all three experiments described below, we test the attenuation hypothesis against the no effect and enhancement hypotheses. We do so by fitting Equation 4 to all groups, regardless of the number of study phase repetitions, and observing whether for the $4 \mathrm{x}$ and $8 \mathrm{x}$ study groups the observed TE is smaller than, equal to, or larger than, the prediction of that equation. Based on extensive prior model fitting outcomes, we expected Equation 4 to fit very well to the data from the $1 \mathrm{x}$ study groups, making it an appropriate reference for inference about the $4 \mathrm{x}$ and $8 \mathrm{x}$ study 
groups. The design of Experiment 3 will also allow for direct assessment of the correct quantitative predictions of the dual-memory model for the 4x study case.

\section{Experiment 1}

In Experiment 1 we used a design with two independent study repetition groups. The $1 \mathrm{x}$ study group received one exposure for each item during the study phase, and the $4 \mathrm{x}$ study group received 4 exposures for each item. The mechanistically correct dual memory prediction for the 1x study group (Equation 4) was calculated for each participant in both the 1x and 4x study groups, using the $\mathrm{PC}_{\mathrm{R}}$ for each participant in each group to generate the respective $\mathrm{PC}_{\mathrm{T}}$ predictions. The dual-memory model predicts TE attenuation in the $4 \mathrm{x}$ group, such that the observed TE is smaller than predicted by Equation 4 when applied to that group. The enhancement hypothesis, on the other hand, would be consistent with a TE for the $4 \mathrm{x}$ group that is larger than predicted by Equation 4. Finally, a finding that Equation 4 fits as well to the $4 \mathrm{x}$ study group as it does the the $1 \mathrm{x}$ study group would be consistent with the no effect hypothesis, which posits that the efficacy of testing relative to restudy is independent of (i.e., not moderated by) the amount of study phase learning. This approach of comparing the $4 \mathrm{x}$ results to the well-established results for the 1x case (by way of the fit of Equation 4) yields inference that is not dependent on the relative magnitude of the TE in the $1 \mathrm{x}$ and $4 \mathrm{x}$ groups.

\section{Methods}

\section{Participants}

We recruited 95 in lab participants from the University of California, San Diego. The mean age of participants was $20.26(2.11)$.

\section{Materials, design and procedure}


A cued-recall paradigm was used with 80 English word-pairs. Forty of those pairs were identical to those used by Rickard and Pan (2020). Forty additional pairs were selected from normative data using the same criteria described in Rickard and Pan. Word pairs were presented horizontally at the center of the computer monitor, with left- or right-side word placement held constant over repetitions. On all test trials, the left-side word was the cue.

Participants were randomly assigned to either the $1 \mathrm{x}$ or $4 \mathrm{x}$ study group. In session 1 , participants first studied all 80 word-pairs for 6 seconds per trial, either once or four times depending on group assignment. During the immediately following training phase, half of the words were assigned to the restudy condition and half to the cued-recall testing with feedback condition (henceforth, test condition), and those two training tasks were manipulated within-participant. In the final test phase, all words were tested through cued-recall with no feedback. Within each phase, the presentation order of word-pairs or cue-words was randomized independently for each participant. In the training phase, the assignment of word-pairs to restudy or testing was randomized once, and then counterbalanced across participants. In the restudy condition, participants restudied half of the word-pairs for $6 \mathrm{~s}$ each. In the test condition, the cue-word was presented for 5 seconds, within which time the participants were asked to type the corresponding answer. Both the cue-word and the correct target were then presented for 1 second, constituting feedback. During the training phase in this and subsequent experiments, there was only one trial per word-pair in both the restudy and test conditions, yielding 80 total trials, with restudy and test trials randomly mixed. There was a 48-hour retention interval between session 1 and 2. During session 2, the final test phase was administered, involving one cued-recall test per item with no feedback. If a participant was unable to recall the target-word, they were allowed to advance to the next cue-word without making a response. The experiment 
was programmed using E-Prime 2.0 and was run in our laboratory. All materials are accessible at the Open Science Framework (OSF.IO/QSRVD).

\section{Results and discussion}

In the training phase, the mean test condition proportions correct were 0.217 and 0.55 in the $1 \mathrm{x}$ and $4 \mathrm{x}$ groups respectively, $t(93)=3.16, p<0.005$. That result confirms that the study phase repetition manipulation had the expected effect on learning and performance.

Final test results for proportion correct means, as well as the Equation 4 predictions for the test conditions, are shown in Figure 1. A mixed factors ANOVA on the observed proportion correct, with factors of Training Task (restudy vs. test, within participants) and Study Repetition $(1 \mathrm{x}$ vs $4 \mathrm{x}$, between), produced a significant (at alpha $=.05)$ main effect of Training Task, $F(1$, $93)=209.08, p<0.0001$, and a significant main effect of Study Repetition, $F(1,93)=18.1, p<$ 0.0001. There was no evidence for an interaction between those two factors, $F(1,93)=0.0064, p$ $=0.91$. In the restudy condition, mean proportion correct was 0.238 and 0.411 in the $1 \mathrm{x}$ and $4 \mathrm{x}$ groups respectively, $t(93)=-4.07, p<0.0001$. In the test condition, mean proportion correct was 0.404 and 0.601 in the $1 \mathrm{x}$ and $4 \mathrm{x}$ groups respectively, $t(93)=-4.13, p<0.0001$.

We next evaluated the Equation 4 predictions for mean proportion correct. A mixed factors ANOVA with factors of Data Type (observed vs. predicted $\mathrm{PC}_{\mathrm{T}}$, within; restudy data were excluded from this analysis) and Study Repetition yielded a non-significant main effect of Data Type, $F(1,93)=0.51, p=0.48$, and a non-significant interaction between Data Type and Study Repetition $F(1,93)=0.91, p=0.34$. To further evaluate the null hypothesis that the Equation 4 prediction matches the data, a Bayes factor was calculated for both the Study Repetition contrasts. The $\mathrm{r}$ (prior) value was set to 0.0707 for all tests. The Bayes factor for both the $1 \mathrm{x}$ group $\left(B F_{01}=5.97\right)$ and the $4 x$ group $\left(B F_{01}=5.16\right)$ favored the null hypothesis. 
The cumulative distribution results for $\mathrm{PC}_{\mathrm{T}}$ are shown in Figure 2. Equation 4 fitted well overall for both the $1 \mathrm{x}$ and $4 \mathrm{x}$ study groups, with mean absolute deviations (MAD) between predicted and observed values over quantiles of 0.04 in the $1 \mathrm{x}$ study group and 0.047 in the $4 \mathrm{x}$ group. Given the relatively small sample sizes, the modest observed deviations from the distribution predictions are expected even if the Equation 4 holds to a close approximation. In Rickard (2020), the model fitted very well to the distribution for a dataset of 509 participants combined over experiments, but exhibited similar localized, idiosyncratic distribution fit deviations when fitted to individual datasets with sample sizes approaching those of the current study.

To conclude, the dual memory model Equation 4 prediction fitted equivalently well to the data from the $1 \mathrm{x}$ and $4 \mathrm{x}$ study groups. These results are consistent with the no change hypothesis; it appears that the efficacy of testing over restudy is independent of the amount of prior study-based learning, at least in the current experimental case. The results are inconsistent with the enhancement hypothesis, the attenuation hypothesis, and the dual-memory model, which predicts testing effect attenuation in the $4 \mathrm{x}$ study case relative to the Equation 4 prediction. 


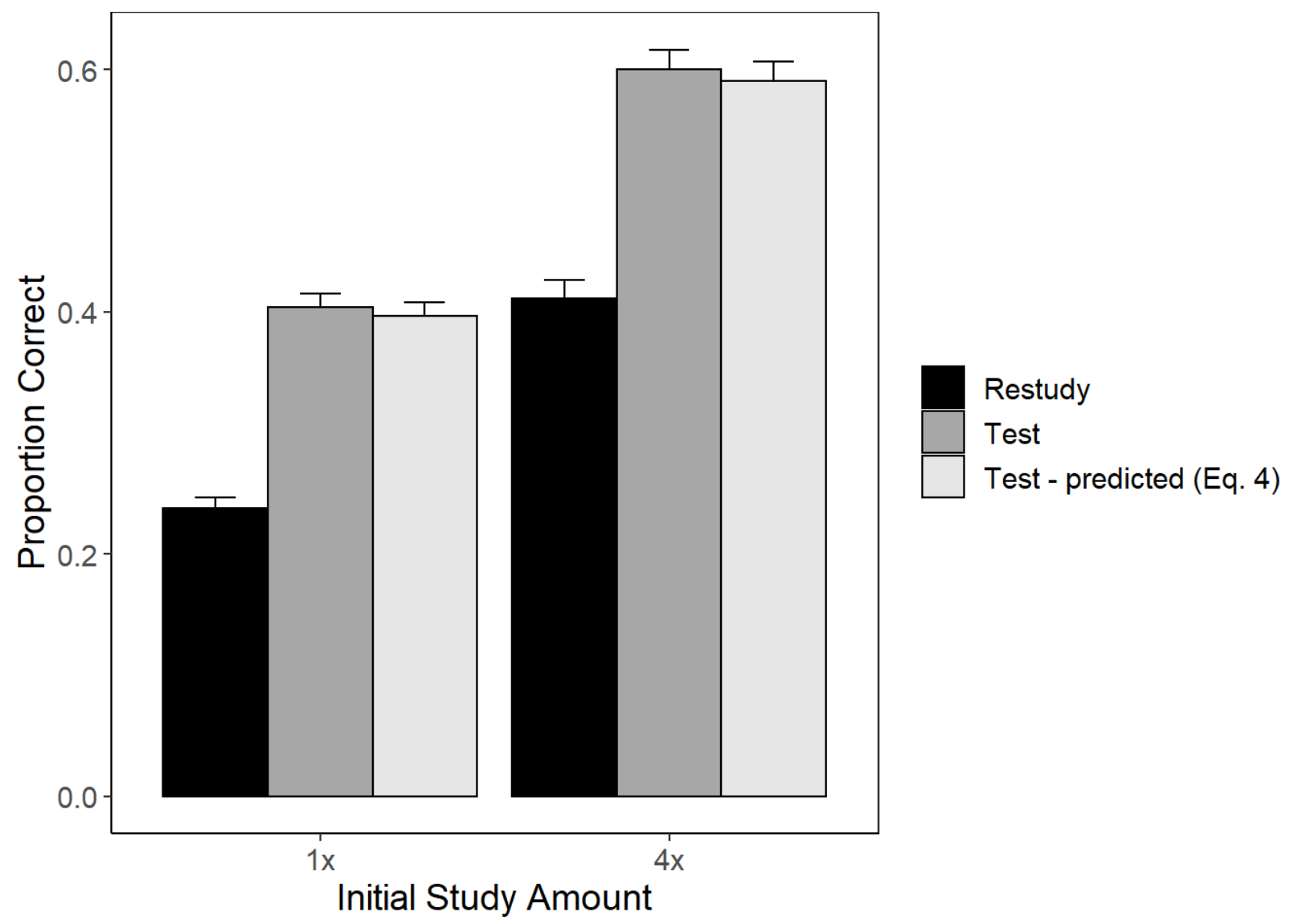

Figure 1. Final test means along with dual-memory model predictions for the test condition in each initial study group. Error bars are standard errors calculated separately for each condition and group. 

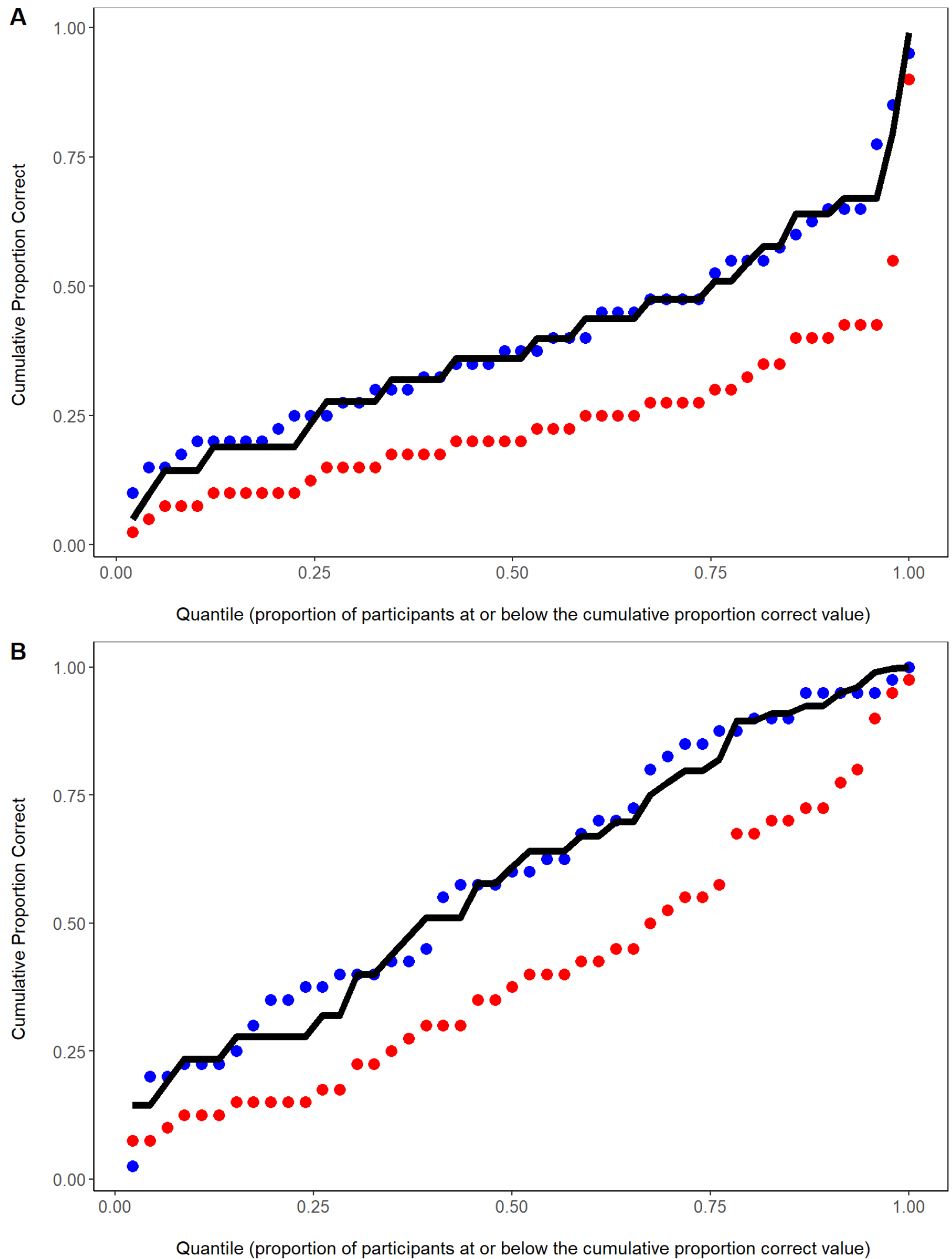

- Restudy - Test $\square$ Test - predicted (Eq. 4) 
Figure 2. Cumulative distribution plots for both initial study conditions. In both conditions, the model closely predicts the cumulative distribution of the test condition.

\section{Experiment 2}

In Experiment 2 we explored whether the results of Experiment 1 extend to a higher level of study repetition. One group received $4 \mathrm{x}$ study repetitions, replicating the $4 \mathrm{x}$ group of Experiment 1, and the other group received 8x study repetitions.

\section{Methods}

\section{Participants}

We recruited 150 participants at the University of California, San Diego for this online experiment. Thirteen participants did not return for session 2, 7 participants erroneously completed only session 2 , and 1 participant was removed because they previously completed a different experiment with the same stimuli. This left 129 participants whose data were analyzed. Participants had a mean age of 20.69 (2.384).

\section{Materials, design and procedure}

Experiment 2 is identical to Experiment 1, except that the experiment was run online, the number of items was reduced from 80 to 40 (20 in each training phase condition; 20 in each final test condition), and the repetition groups were $4 x$ and $8 x$. The number of items was reduced to 40 so that participants in the $8 \mathrm{x}$ could complete session 1 in less than one hour. Participants were randomly assigned into one of two groups of study: 4x or $8 x$.

The experiment was coded in JS, HTML, and CSS. The jsPsych library (de Leeuw, 2015) was used for randomization of the training condition and the word-pair order. Participants were emailed a link to session 1 . They had a 12-hour window to complete it. Forty-eight hours after completing session 1, participants were emailed a reminder to perform session 2 and had a 12-hour window to do so. The $4 \mathrm{x}$ and $8 \mathrm{x}$ groups had average retention intervals of $48.55(S D=$ 
6.20) and 49.39 (12.32) hours. There was no significant difference of retention interval between the $4 x$ and $8 x$ groups, $t(127)=-0.48, p=0.63$.

\section{Results and Discussion}

In the training phase, participants had a mean proportion correct on test trials of 0.463 and 0.600 in the $4 x$ and $8 x$ groups respectively, $t(127)=-3.16, p<0.005$. Final test results for mean proportion correct are shown in Figure 3. A mixed factors ANOVA revealed a main effect of Training Task, $F(1,127)=112.88, p<0.0001$ and a main effect of Study Repetition, $F(1,127)$ $=8.28, p<0.005$. There was no interaction between those factors, $F(1,93)=0.015, p=0.9$. For items that were trained through restudy, participants had a mean proportion correct of 0.389 and 0.513 in the $4 \mathrm{x}$ and $8 \mathrm{x}$ groups respectively, $t(127)=-2.66, p<0.01$. In the final testing phase for items that were trained through recall, participants had a mean proportion correct of 0.560 and 0.685 in the $4 \mathrm{x}$ and $8 \mathrm{x}$ groups respectively, $t(127)=-2.77, p<0.01$.

In the mixed factors ANOVA for assessing the fit of Equation 4 (identical to that in Experiment 1), there was again a non-significant effect of Data Type $F(1,127)=0.049, p=0.83$ and a non-significant interaction, $F(1,127)=0.059, p=0.81$. Bayes factor results for Data Type for the $4 \mathrm{x}$ group $\left(B F_{01}=6.92\right)$ and for the $8 \mathrm{x}$ group $\left(B F_{01}=7.3\right)$ favored the null hypothesis. Equation 4 also fitted well to the cumulative distribution, regardless of the number of study repetitions (Figure 4), with MAD values of 0.035 and 0.027 in the $4 x$ and $8 x$ groups respectively. To conclude, results of this experiment again speak against the attenuation and enhancement hypotheses, and in favor of the no change hypothesis. The results so far support the conclusion that the efficacy of testing relative to restudy is independent of the amount of prior study. 


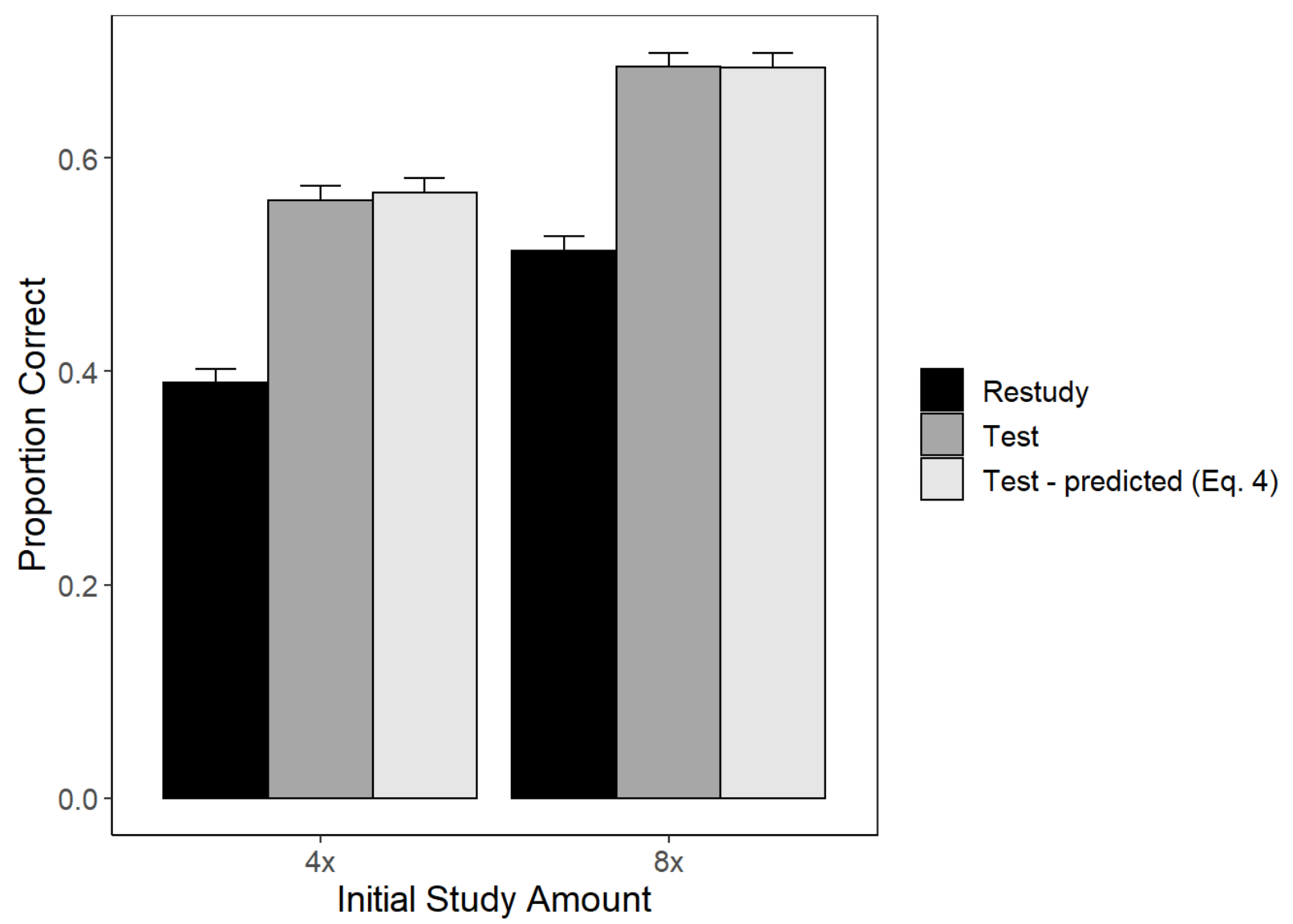

Figure 3. Final test means along with dual-memory model predictions for the test condition in each initial study group. Error bars are standard errors calculated separately for each condition and group. 

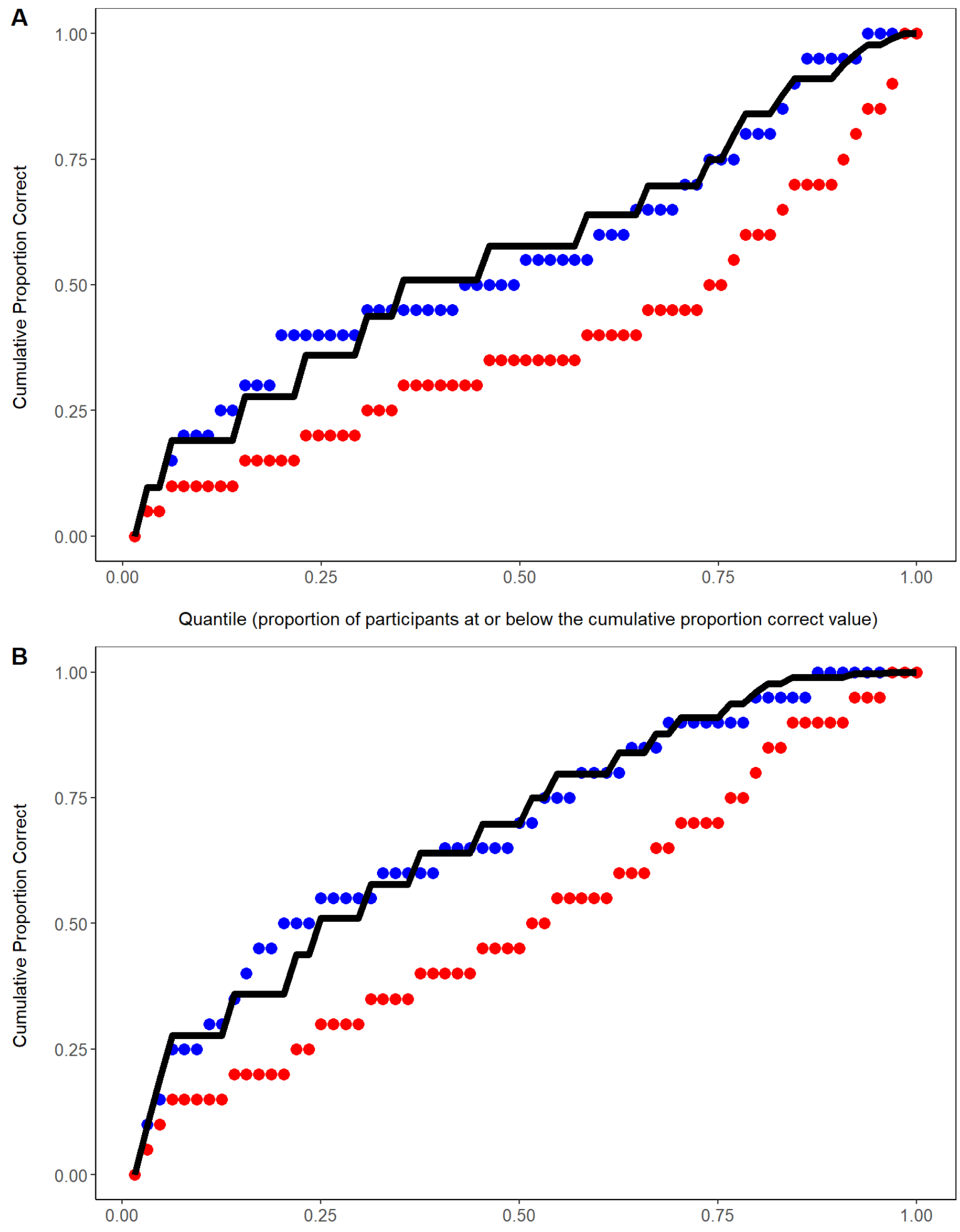

Quantile (proportion of participants at or below the cumulative proportion correct value)

- Restudy - Test - Test - predicted (Eq. 4) 
Figure 4. Cumulative distribution plots for both initial study conditions. In both conditions, the model closely predicts the cumulative distribution of the test condition.

\section{Experiment 3}

A limitation of Experiments 1 and 2 is that the between-group manipulation of study repetition did not allow the correct quantitative dual-memory model prediction for the multi-study case to be calculated, and thus a direct inferential test of that model's predictions for the $4 \mathrm{x}$ and $8 \mathrm{x}$ study groups could not be conducted. The within-participant manipulation of both study repetition and training task in the current experiment does allow for calculation of the correct model prediction for the multi-study case. To review, according to the model, learning through study and through testing create independent study and test memories. Hence, the model estimate for proportion correct through test memory on the final test is the same for the $1 \mathrm{x}$ and 4 study repetition conditions that were used in this Experiment (given the random item assignment and the fully within participant manipulations); i.e., there is only one training phase test trial in each of those conditions, and hence the model predicts stochastically equivalent test memory strength after training in those conditions. In contrast, study memory strength for both restudied items and tested items will be greater in the $4 \mathrm{x}$ study condition than in the $1 \mathrm{x}$ study condition. The mechanistically correct dual-memory model prediction for $\mathrm{PC}_{\mathrm{T}}$ in the $4 \mathrm{x}$ condition must accommodate that fact. As for the $1 \mathrm{x}$ study case, model predictions for $\mathrm{PC}_{\mathrm{T}}$ in the multistudy case depend only on observed $\mathrm{PC}_{\mathrm{R}}$. However, the separate $\mathrm{PC}_{\mathrm{R}}$ values from both the $1 \mathrm{x}$ and $4 \mathrm{x}$ restudy conditions are needed for the calculation. The necessary calculations were illustrated for an example case in the Introduction. More formally, for the 4x study condition of the current experiment, the general prediction equation of the model,

$$
\mathrm{P}_{\mathrm{T}}=\mathrm{P}_{\mathrm{T}-\mathrm{s}}+\mathrm{P}_{\mathrm{T}-\mathrm{t}}-\mathrm{P}_{\mathrm{T}-\mathrm{S}} \mathrm{P}_{\mathrm{T}-\mathrm{t}}
$$

cannot be reduced to Equation 4, but instead is written as, 


$$
\mathrm{PC}_{\mathrm{T}(4 \mathrm{x})}=\mathrm{PC}_{\mathrm{R}(4 \mathrm{x})}+\mathrm{PC}_{\mathrm{R}(1 \mathrm{x})}-\mathrm{PC}_{\mathrm{R}(4 \mathrm{x})} \mathrm{PC}_{\mathrm{R}(1 \mathrm{x}),}
$$

where $\mathrm{PC}_{\mathrm{R}(1 \mathrm{x})}$ and $\mathrm{PC}_{\mathrm{R}(4 \mathrm{x})}$ are the observed final test restudy proportions correct in the $1 \mathrm{x}$ and $4 \mathrm{x}$ study conditions, respectively. Only in the fully within-participant design of Experiment 3 are both $\mathrm{PC}_{\mathrm{R}(1 \mathrm{x})}$ and $\mathrm{PC}_{\mathrm{R}(4 \mathrm{x})}$ available for each participant.

\section{Methods}

\section{Participants}

We recruited 67 participants at the University of California, San Diego for this online experiment. Participants signed up for the study online through our Psychology Department participant pool, and each participant was emailed a link to initiate session 1. Participants were told they had a 12-hour window to initiate the experiment. Forty-eight hours after session 1, participants were emailed a reminder to initiate session 2, and they were informed that they had a 12-hour window to complete that session and to receive credit. The average retention interval was 45.63(9.04) hrs.

Fifteen participants did not return for session 2. One participant was removed because they had a retention interval of about 900 hours. This left 51 participants whose data were analyzed. Participants had a mean age of 20.56 (1.81).

\section{Materials, design and procedure}

The design of Experiment 3 is the same as that of Experiment 1, with the exception that all of the conditions are within-participant and that the experiment was conducted online (using the same methods as described for Experiment 2). That fully within design yielded four crossed conditions: restudy with $1 \mathrm{x}$ study, testing with $1 \mathrm{x}$ study, restudy with $4 \mathrm{x}$ study, and testing with 4x study. The 80 items from the first experiment were again used, but because all four conditions were within-participant in this experiment, the intended number of items per condition was 20 
instead of 40 . There was an error in the program code that randomly assigned items to conditions. Specifically, there was no constraint in the code to assign exactly 20 items to each condition. Over participants, actual item assignment per condition ranged from 13 to 27 items. Averaged over participants, the average number of items assigned was as follows: $1 \mathrm{x}$ restudy (20.06), $4 \mathrm{x}$ restudy (19.94), $1 \mathrm{x}$ tested (19.94), $4 \mathrm{x}$ tested (20.06). The programming error was limited to the number of items randomly assigned to each condition during the training phase, and it should not materially affect the results.

\section{Results and Discussion}

In the training phase, the mean proportion correct on test trials was 0.231 and 0.510 in the $1 \mathrm{x}$ and $4 \mathrm{x}$ conditions respectively, $t(50)=5.73, p<0.0001$. Final test results are summarized in Figure 5. A repeated measures ANOVA on the observed proportion correct revealed significant main effects of both Training Task, $F(1,50)=77.64, p<0.0001$, and Study Repetition, $F(1,50)$ $=63.82, p<0.0001$. There was no interaction between those two factors, $F(1,50)=0.65, p=$ 0.42. Limited to items that were trained through restudy, the mean proportion correct was 0.286 and 0.429 in the $1 \mathrm{x}$ and $4 \mathrm{x}$ groups, respectively, $t(50)=-5.76, p<0.001$. Limited to items trained through testing, the mean proportion correct was 0.434 and 0.60 in the $1 \mathrm{x}$ and $4 \mathrm{x}$ groups respectively, $t(50)=-7.37, p<0.0001$.

A repeated measures ANOVA was also conducted to assess the fit of Equation 4. There were again non-significant effects for both Data Type, $F(1,50)=0.42, p=0.52$ and the Data Type by Study Repetition interaction, $F(1,50)=0.087, p=0.77$. The Bayes factors for Data Type in both the $1 \mathrm{x}$ condition $\left(B F_{01}=6.26\right)$ and for the $4 \mathrm{x}$ condition $\left(B F_{01}=5.4\right)$ again favored the null hypothesis. 
Next we tested the mechanistically correct dual-memory model prediction for $\mathrm{PC}_{\mathrm{T}}$ in the 4x study condition (using Equation 6; see the light gray bar to the far right in Figure 5). That prediction underestimated the observed $\mathrm{PC}_{\mathrm{T}}$ by $0.068, t(50)=-3.02, p=0.01$, whereas the Equation 4 has an error of only $0.016, t(50)=-0.64, p=0.52$. In summary, the results for mean proportion correct match those of Experiment 1 and 2, supporting the no effect hypothesis and speak against the enhancement hypothesis, the attenuation hypothesis, and the dual-memory model prediction for the multistudy case.

The cumulative test condition proportions correct, along with the predictions of Equation 4, are shown in Figure 6, with MAD values 0.069 and 0.070 in the $1 \mathrm{x}$ and $4 \mathrm{x}$ conditions, respectively. Those systematically poorer distribution fits to both repetition conditions are surprising in light of the good fits of Equation 4 to the mean proportions correct in all three experiments and to the proportion correct distributions in Experiments 1 and 2 (and also to separate data fits in Rickard \& Pan, 2018, and Rickard, 2020). That anomalous result conceivably reflects random factors. Alternatively, it may reflect an unanticipated interaction between the within participant manipulation of study repetition (i.e., the intermixing $1 \mathrm{x}$ and $4 \mathrm{x}$ repetition item) and individual differences in task ability, which as noted earlier appear to explain a major component of the proportion correct variability over participants in this paradigm (Rickard, 2020). Participants may have been aware of the uneven number of study repetitions over items and that may have affected learning in a way that interacted with individual differences, although we cannot advance a specific process account. In any case, it appears that, in both study repetition conditions, participants with lower overall task ability (i.e., participants with results on the lower half of the distribution in Figure 6) benefited somewhat more from testing than Equation 4 predicts and that participants with higher overall task ability (i.e., on the 
right half of Figure 6) benefitted somewhat less from testing than Equation 4 predicts.

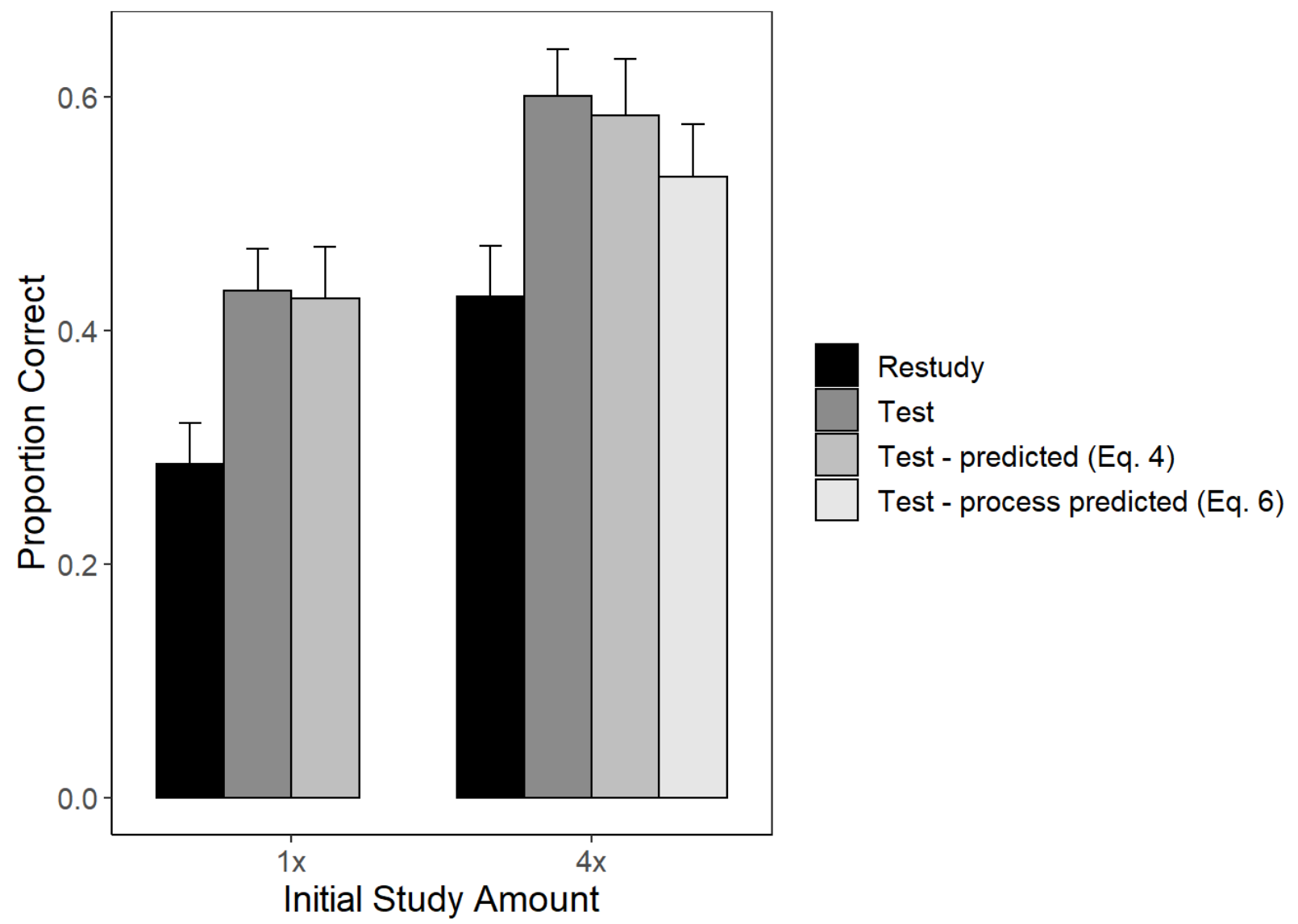

Figure 5. Final test means along with dual-memory model prediction for the test condition in each initial study group and the process model prediction in the $4 \mathrm{x}$ study group. Error bars are standard errors calculated separately for each condition and group. 

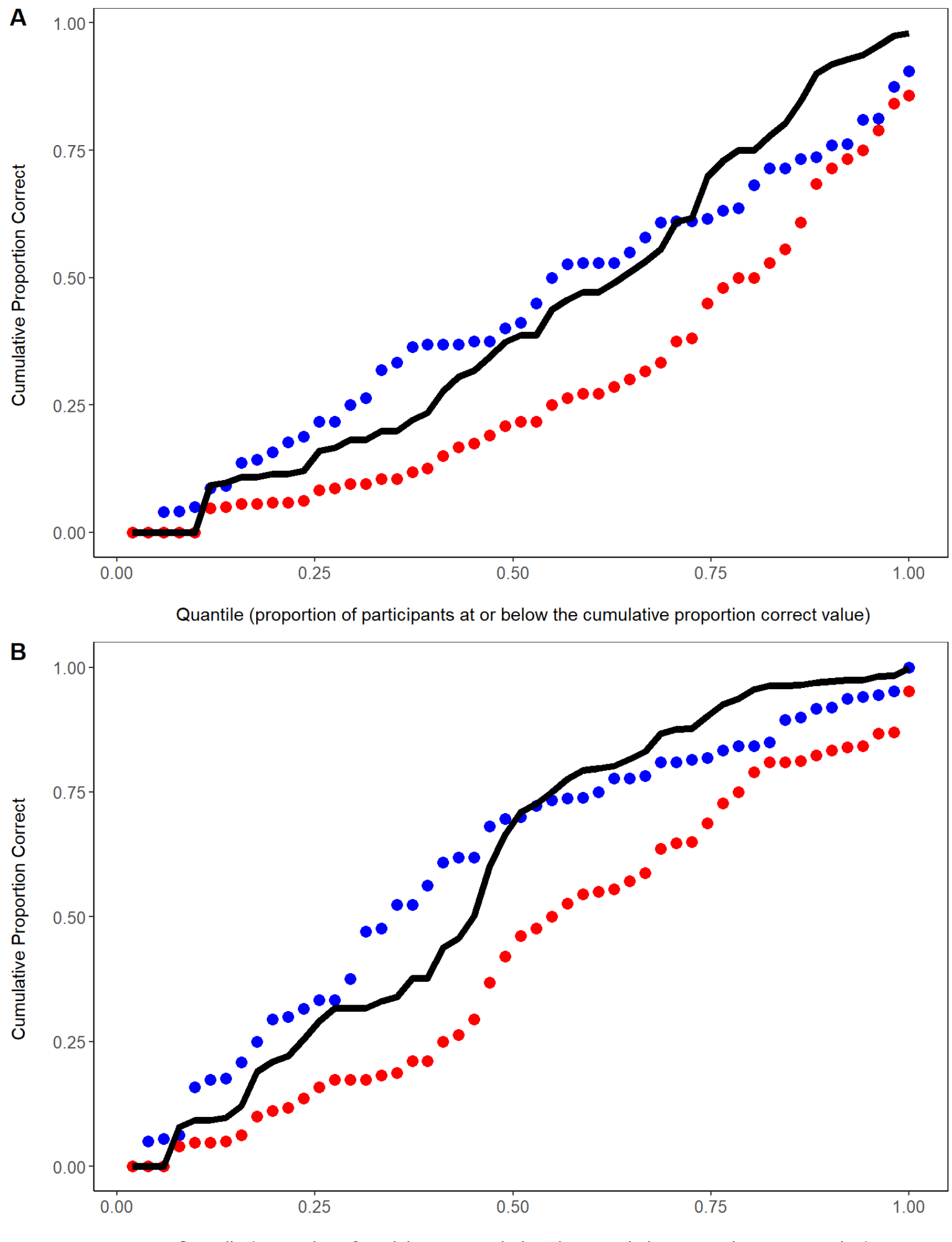

Quantile (proportion of participants at or below the cumulative proportion correct value)

- Restudy - Test $\square$ Test - predicted (Eq. 4) 
Figure 6. Cumulative distribution plots for both initial study conditions.

\section{General Discussion}

In this study we explored the effect of prior knowledge of to be learned materials - as manipulated through variation in the number of study phase item repetitions - on the efficacy of testing. Three hypotheses were considered as the level of prior knowledge becomes greater: attenuation of the testing effect, enhancement of it, and no effect. According to the attenuation hypothesis and its instantiation in the dual-memory model, the efficacy of a test relative to restudy should be a decreasing function of prior study-based learning. Across three experiments, no evidence for that hypothesis was observed. There was also no evidence for the enhancement hypothesis in any of the three experiments. Instead, the results overall were consistent with the no effect hypothesis, according to which the efficacy of a test relative to restudy is unrelated to the amount of prior study.

Although restudy proportion correct increased with increasing study repetition (as expected), that fact does not compromise the inferences that can be drawn from the application of Equation 4, because that equation has fitted well to the $1 \mathrm{x}$ study case across a variety of restudy proportion correct values for both experimental means (Rickard \& Pan, 2018) and proportion correct distributions (Rickard,2020), as well as to the three experiments of the current paper (with the exception of the distribution results for Experiment 3). Also, the viability of our conclusions based on application of Equation 4 does not depend on whether the process assumptions of the dual-memory model are correct. For current purposes, Equation 4 is useful for testing the three hypotheses based solely on the empirical fact that it fits a large corpus of data so well for the $1 \mathrm{x}$ study case. 
The conclusion that testing effect efficacy is independent of prior study for cued-recall may hold even under circumstances in which proportion correct in the restudy and test conditions approaches one and the testing effect magnitude by that measure approaches zero. There is no principled reason to expect the difference in underlying learning for those two tasks to be reduced when proportion correct reaches the ceiling. Indeed, it is likely that final test proportions correct in that hypothetical experiment could be reduced to virtually any desired value by merely increasing the retention interval, and Equation 4 may still predict the testing effect magnitude in that case. Our finding that Equation 4 has held for retention intervals varying between 5 minutes and one week (Rickard, 2020) buttresses that speculation.

\section{Theoretical Considerations}

The current results appear to rule-out the dual-memory model in its current form as an account of the effects of study phase repetition on the TE. One way to adjust that model to account for the current results is to assume that, despite the prior claims in the literature to the contrary (Kornell, Klein, \& Rawson, 2015; Rickard, 2020), learning on a training test trial with feedback is greater when the correct answer is retrieved than when it is not. If that is true, it may offset the attenuation that would otherwise be expected in the dual-memory model, such that Equation 4 holds even for the $4 x$ and $8 x$ repetition cases. It remains to be determined, however, whether that equation would hold in that scenario while preserving other core properties and assumptions of the model. A second possibility is that test memory strength is always similar to study memory strength, regardless of the number of prior study phase repetitions. In that account, a stronger study memory promotes a stronger test memory, even though those memories are separate. In that case Equation 4 may still hold regardless of the level of prior study knowledge. That version of the model may be less appealing than the current version, however, 
because the assumption that study and test memory are independent would no longer hold with respect to the degree of prior study learning, yet would be maintained with respect to the relation between study and test memory strengths for tested items.

Alternatively, the claim of two retrieval routes in the dual-memory model may be incorrect, and instead both restudy and test trials strengthen a single route to retrieval that is established during the study phase. In that account, memory strengthening on a test trial with feedback is greater than memory strengthening on a restudy trial by some mechanism to be specified (as an example of a single route account in the literature for the case of testing without feedback, see the Bifurcation model; Kornell, Bjork, and Garcia, 2011). It remains to be seen whether a single-route psychological process theory can accommodate the broader set of results that are accounted for by the dual-memory model and by Equation 4. Other theoretical approaches may also have merit.

\section{Conclusions}

The current results make several new contributions to research on the testing effect. First, they constitute the first evidence that the level of prior content knowledge does not substantially moderate the efficacy of testing relative study, or restudy. That result remains to be generalized beyond the current experimental design, but it is reasonable to expect it to hold at least for the general case of cued-recall testing. Second, Equation 4 now describes the broadest and best supported empirical regularity in the testing effect literature to date. Even though that regularity will have limits, it is likely in our view to remain a crucible for theory development, particularly in light of the experimental paradigm that we have used, which is minimalist and (we believe) effectively distills the effecting effect, excluding extraneous factors that may moderate outcome. Third, the current results reveal a clear limit to the applicability of the dual-memory model of 
test-enhanced learning. Finally, the results suggest that testing can be applied at various levels of content mastery in applied settings without risk of diminished potency. 


\section{References}

Abel, M., \& Roediger, H. L. (2017). Comparing the testing effect under blocked and mixed practice: The mnemonic benefits of retrieval practice are not affected by practice format. Memory and Cognition, 45(1), 81-92. https://doi.org/10.3758/s13421-016-0641-8

Carpenter, S. K., \& DeLosh, E. L. (2005). Application of the testing and spacing effects to name learning. Applied Cognitive Psychology, 19(5), 619-636.

Carpenter, S. K., \& Delosh, E. L. (2006). Impoverished cue support enhances subsequent retention: Support for the elaborative retrieval explanation of the testing effect. Memory, 34(2), 268-276.

Carpenter, S. K., Pashler, H., Wixted, J. T., \& Vul, E. (2008). The effects of tests on learning and forgetting. Memory and Cognition, 36(2), 438-448.

Carpenter, S. K., Cepeda, N. J., Rohrer, D., Kang, S. H. K., \& Pashler, H. (2012). Using Spacing to Enhance Diverse Forms of Learning: Review of Recent Research and Implications for Instruction. Educational Psychology Review, 24(3), 369-378. https://doi.org/10.1007/s10648-012-9205-z

Cepeda, N. J., Pashler, H., Vul, E., Wixted, J. T., \& Rohrer, D. (2006). Distributed practice in verbal recall tasks: A review and quantitative synthesis. Psychological Bulletin, 132(3), 354-380. https://doi.org/10.1037/0033-2909.132.3.354

Cepeda, N. J., Vul, E., Rohrer, D., Wixted, J. T., \& Pashler, H. (2008). Spacing effects in learning: A temporal ridgeline of optimal retention. Psychological Science, 19(11), 1095-1102. https://doi.org/10.1111/j.1467-9280.2008.02209.x

Cull, W. L. (2000). Untangling the Benefits of Multiple Study Opportunities and Repeated Testing for Cued-recall. Applied Cognitive Psychology, 14(3), 215-235. 
de Leeuw, J. R. (2015). jsPsych: A JavaScript library for creating behavioral experiments in a web browser. Behavior Research Methods, 47(1), 1-12.

Foster, N. L., Mueller, M. L., Was, C., Rawson, K. A., \& Dunlosky, J. (2019). Why does interleaving improve math learning? The contributions of discriminative contrast and distributed practice. Memory and Cognition, 47(6), 1088-1101.

\section{https://doi.org/10.3758/s13421-019-00918-4}

Kang, S. H. K., McDermott, K. B., \& Roediger, H. L. (2007). Test format and corrective feedback modify the effect of testing on long-term retention. European Journal of Cognitive Psychology, 19(4-5), 528-558. https://doi.org/10.1080/09541440601056620

Karpicke, J. D., \& Blunt, J. R. (2011). Retrieval practice produces more learning than elaborative studying with concept mapping. Science, 331(6018), 772-775.

Karpicke, J. D., Lehman, M., \& Aue, W. R. (2014). Retrieval-Based Learning. An Episodic Context Account. In Psychology of Learning and Motivation - Advances in Research and Theory (Vol. 61). https://doi.org/10.1016/B978-0-12-800283-4.00007-1.

Kornell, N., Bjork, R. A., \& Garcia, M. A. (2011). Why tests appear to prevent forgetting: A distribution-based bifurcation model. Journal of Memory and Language, 65(2), 85-97.

Kornell, N., \& Vaughn, K. E. (2016). How Retrieval Attempts Affect Learning. Psychology of Learning and Motivation, 65, 183-215. https://doi.org/10.1016/bs.plm.2016.03.003

Kromann, C. B., Jensen, M. L., \& Ringsted, C. (2009). The effect of testing on skills learning. Medical Education, 43(1), 21-27. https://doi.org/10.1111/j.1365-2923.2008.03245.x

Lantz, M. E. (2010). The use of "Clickers" in the classroom: Teaching innovation or merely an amusing novelty? Computers in Human Behavior, 26(4), 556-561. 
Larsen, D. P., Butler, A. C., \& Roediger, H. L. (2009). Repeated testing improves long-term retention relative to repeated study: A randomised controlled trial. Medical Education, 43(12), 1174-1181.

Latimier, A., Riegert, A., Peyre, H., Ly, S. T., Casati, R., \& Ramus, F. (2019). Does pre-testing promote better retention than post-testing?. NPJ science of learning, 4(1), 1-7.

McDaniel, M. A., Anderson, J. L., Derbish, M. H., \& Morrisette, N. (2007). Testing the testing effect in the classroom. European Journal of Cognitive Psychology, 19(4-5), 494-513.

Mozer, M. C., Pashler, H., Cepeda, N., Lindsey, R., \& Vul, E. (2009). Predicting the optimal spacing of study: A multiscale context model of memory. Advances in Neural Information Processing Systems 22 - Proceedings of the 2009 Conference, 1321-1330.

Nungester, R. J., \& Duchastel, P. C. (1982). Testing versus review: Effects on retention. Journal of Educational Psychology, 74(1), 18-22.

Pan, S. C., Cooke, J., Little, J. L., McDaniel, M. A., Foster, E. R., Connor, L. T., \& Rickard, T. C. (2019). Online and clicker quizzing on jargon terms enhances definition-focused but not conceptually focused biology exam performance. CBE_Life Sciences Education, 18(4), $\operatorname{ar} 54$.

Pan, S. C., \& Rickard, T. C. (2018). Transfer of test-enhanced learning: Meta-analytic review and synthesis. Psychological Bulletin, 144(7), 710-756.

Pan, S. C., \& Sana, F. (2021). Pretesting versus posttesting: Comparing the pedagogical benefits of errorful generation and retrieval practice. Journal of Experimental Psychology: Applied.

Pan, S. C., Tajran, J., Lovelett, J., Osuna, J., \& Rickard, T. C. (2019). Does Interleaved Practice Enhance Foreign Language Learning? The Effects of Training Schedule on Spanish Verb 
Conjugation Skills. Journal of Educational Psychology, 1-17. https://doi.org/10.1037/edu0000336.

Pashler, H., Cepeda, N. J., Wixted, J. T., \& Rohrer, D. (2005). When Does Feedback Facilitate Learning of Words? Journal of Experimental Psychology: Learning, Memory, and Cognition, 31(1), 3-8.

Roediger, H. L. \& Karpicke, J. D. (2006). Psychological Science, 17 (3), 2490255.

Richland, L. E., Kornell, N., \& Kao, L. S. (2009). The pretesting effect: Do unsuccessful retrieval attempts enhance learning?. Journal of Experimental Psychology: Applied, 15(3), 243.

Rickard, T. C., \& Pan, S. C. (2018). A dual memory theory of the testing effect. Psychonomic Bulletin and Review, 25(3), 847-869.

Rickard, T. C., \& Pan, S. C. (2020). Test-enhanced learning for pairs and triplets: When and why does transfer occur?. Memory \& Cognition, 48, 1146-1160.

Rohrer, D. (2012). Interleaving Helps Students Distinguish among Similar Concepts. Educational Psychology Review, 24(3), 355-367. https://doi.org/10.1007/s10648-012-9201-3.

Rohrer, D. (2015). Student Instruction Should Be Distributed Over Long Time Periods. Educational Psychology Review, 27(4), 635-643. https://doi.org/10.1007/s10648-015-9332-4.

Rohrer, D., Dedrick, R. F., Hartwig, M. K., \& Cheung, C. N. (2020). A randomized controlled trial of interleaved mathematics practice. Journal of Educational Psychology, 112(1), 40-52. https://doi.org/10.1037/edu0000367. 
Rowland, C. A. (2014). The effect of testing versus restudy on retention: a meta-analytic review of the testing effect. Psychological Bulletin, 140(6), 1432-1463.

van den Broek, G., Takashima, A., Wiklund-Hörnqvist, C., Karlsson Wirebring, L., Segers, E., Verhoeven, L., \& Nyberg, L. (2016). Neurocognitive mechanisms of the "testing effect": A review. Trends in Neuroscience and Education, 5(2), 52-66.

https://doi.org/10.1016/j.tine.2016.05.001. 